\title{
Identificação de marsupiais do Rio Grande do Sul através da microestrutura dos pelos-guarda
}

\author{
Maury Sayão Lobato Abreu ${ }^{1}$, Alexandre Uarth Christoff ${ }^{2}$ \& Emerson Monteiro Vieira ${ }^{3,4}$ \\ ${ }^{1}$ Laboratório de Ecologia de Mamíferos, Zoologia, Universidade do Vale do Rio dos Sinos - UNISINOS, \\ Av. Unisinos, 950, CEP 93022-000, São Leopoldo, RS, Brasil, e-mail: maury.abreu@ gmail.com \\ ${ }^{2}$ Departamento de Biologia, Museu de Ciências Naturais, Universidade Luterana do Brasil - ULBRA, \\ Av. Farroupilha, 8001, Canoas, RS, Brasil,e-mail: auchrist@ulbra.br \\ ${ }^{3}$ Departamento de Ecologia, Instituto de Ciências Biológicas, Universidade de Brasília - UnB, \\ CP 04457, CEP 70919-970, Brasília, DF, Brasil \\ ${ }^{4}$ Autor para correspondência: Emerson Monteiro Vieira, e-mail: emvieira@unb.br
}

\begin{abstract}
ABREU, M.S.L., CHRISTOFF, A.U. \& VIEIRA, E.M. Identification of marsupials from southern Brazil using microstructure of guard-hairs. Biota Neotrop. 11(3): http://www.biotaneotropica.org.br/v11n3/en/ abstract?identification-key +bn03111032011
\end{abstract}

\begin{abstract}
The analysis of mammalian hairs is a non-invasive technique that may help in the identification of Brazilian marsupials. Our objective was to identify microscopic patterns of marsupial guard-hairs (cuticle and medulla) from species with occurrence in the state of Rio Grande do Sul, southern Brazil. We also present a hair-based identification key for this group. Specifically for Monodelphis, a still taxonomically obscure genus that needs revision, we present detailed description of observed hair patterns. We obtained the analyzed material by collecting hair samples of marsupials captured in field or else deposited in different zoological collections. We identified hair patterns of ten marsupial species: Caluromys lanatus, Chironectes minimus, Didelphis aurita, Didelphis albiventris, Cryptonanus guahybae, Gracilinanus agilis, Gracilinanus microtarsus, Lutreolina crassicaudata, Micoureus paraguayanus and Philander frenatus. Individuals of the Monodelphis genus were analyzed together, considered as part of the dimidiata group. We found three distinct patterns for this group, suggesting the possible occurrence of a third species besides the two already recognized (M. brevicaudis and M. dimidiata). These morphs show distinct distribution in the state, with some degree of distribution overlap. The analysis of microscopic patterns of mammal hairs allowed the successful identification of the great majority of marsupials that occur in the Rio Grande do Sul state. Our results suggest that the two Monodelphis species of the dimidiata group might occur with a distinct distribution in the state, one of them occupying mainly the northern part and the other one the eastern region, with some overlap in the central part of the state.
\end{abstract}

Keywords: cuticle, hair identification, identification key, medulla, tricology.

ABREU, M.S.L., CHRISTOFF, A.U. \& VIEIRA, E.M. Identificação de marsupiais do Rio Grande do Sul através da microestrutura dos pelos-guarda. Biota Neotrop. 11(3): http://www.biotaneotropica.org.br/v11n3/ pt/abstract?identification-key+bn03111032011

Resumo: A análise dos padrões microscópicos de pelos de mamíferos é uma técnica não-invasiva que pode auxiliar na identificação dos marsupiais brasileiros. O objetivo deste trabalho foi identificar padrões microscópicos dos pelos-guarda (cutícula e medula) de marsupiais ocorrentes no Rio Grande do Sul (RS), propondo uma chave dicotômica para sua identificação a partir dessas estruturas. Além disso, nós apresentamos, com detalhes, os padrões tricológicos de espécimes do gênero Monodelphis, um gênero ainda taxonomicamente obscuro que requer revisão no RS. Obtivemos o material analisado por meio da coleta de pelos de marsupiais capturados em campo ou provenientes de coleções de diferentes instituições. Identificamos os padrões dos pelos de dez espécies de marsupiais: Caluromys lanatus, Chironectes minimus, Didelphis aurita, Didelphis albiventris, Cryptonanus guahybae, Gracilinanus agilis, Gracilinanus microtarsus, Lutreolina crassicaudata, Micoureus paraguayanus e Philander frenatus. Os indivíduos do gênero Monodelphis foram analisados em conjunto, como parte do grupo dimidiata. Encontramos três padrões dentro desse grupo, sugerindo que possa haver uma terceira espécie além das duas normalmente reconhecidas ( $M$. brevicaudis e $M$. dimidiata). A análise dos padrões microscópicos dos pelos possibilitou a identificação, com sucesso, da grande maioria das espécies à que pertencem os marsupiais do Rio Grande do Sul. Os resultados obtidos sugerem que as duas espécies de Monodelphis grupo dimidiata possam ocorrer com uma distribuição distinta no estado, estando uma presente principalmente na porção norte e a outra na porção leste do RS, com sobreposição na região central.

Palavras-chave: cutícula, identificação por pelos, chave dicotômica, medula, tricologia. 


\section{Introdução}

Os marsupiais da família Didelphidae, ordem Didelphimorphia, distribuem-se atualmente pela região Neotropical, ocupando principalmente a America do Sul e Central. Essa família reúne 17 gêneros e 87 espécies (Gardner 2005), das quais pelo menos 55 ocorrem no Brasil (Rossi et al. 2006). Esses animais exercem importante papel ecológico nos ecossistemas onde ocorrem, podendo atuar como dispersores de sementes (Cáceres et al. 1999, Cáceres \& Monteiro-Filho 2000, Cáceres 2002), polinizadores (Gribel 1988, Vieira et al. 1991), ou como presas para carnívoros (Jácomo et al. 2004, Gatti et al. 2006a, b, Pedó et al. 2006, Abreu et al. 2010).

Embora existam ainda muitas lacunas sobre a distribuição e status taxonômicos de muitas espécies de marsupiais, nos últimos dez anos estudos que avaliam relações filogenéticas ou que discutem composição de gêneros têm sido realizados por distintos grupos a partir de estudos de características anatômicas ou de sequências gênicas (e.g. Patton \& da Silva 1997, Jansa \& Voss 2000, Voss \& Jansa 2003, Voss et al. 2004, Jansa \& Voss 2005, Jansa et al. 2006). Contudo, a falta de estudos realizados no extremo sul do Brasil que incluam espécies de marsupiais dificulta a descrição da riqueza e diversidade deste grupo nessa região, alem de prejudicar discussões de cunho ecológico ou conservacionista (Vieira \& Iob 2003). Por este motivo ainda há algumas dúvidas sobre as espécies de marsupiais que ocorrem no estado do Rio Grande do Sul. Esse cenário é ainda prejudicado pela baixa representatividade desses animais em coleções zoológicas, possivelmente devido à baixa abundância local de algumas espécies ou por sua difícil captura com métodos convencionais. Por exemplo, muitos marsupiais didelfídeos possuem habito arborícola, e por isso raramente são capturados em armadilhas instaladas apenas no solo.

Atualmente, há 10 espécies de marsupiais com ocorrência confirmada no Rio Grande do Sul (segundo Rossi et al. (2006)): Caluromys lanatus (Olfers, 1818), Chironectes minimus (Zimmermann, 1780), Didelphis albiventris (Lund, 1840), Gracilinanus microtarsus (Wagner, 1842), Cryptonanus guahybae (Tate, 1931), Lutreolina crassicaudata (Desmarest, 1804), Micoureus paraguayanus (Tate, 1931), Monodelphis dimidiata (Wagner, 1847), Monodelphis iheringi (Thomas, 1888) e Monodelphis sorex (Hensel, 1872). Além desses, Emmons \& Feer (1990) e Brown (2004) incluem a região como área de ocorrência de Didelphis aurita (Wied-Neuwied, 1826) e há registro de ocorrência de Philander frenatus (Olfers, 1818) no nordeste do estado (Sarti 2009) e de Gracilinanus agilis (Burmeister, 1854) na fronteira do mesmo (Brown 2004, Canevari \& Vaccaro 2007). Dessa maneira, possivelmente ocorrem pelo menos 13 espécies de marsupiais no Rio Grande do Sul.

Especificamente em relação ao gênero Monodelphis, grupo de didelfídeos composto por pelo menos 18 espécies que se distribuem amplamente na América do Sul (Gardner 2005), a determinação de quais espécies de fato ocorrem no Rio Grande do Sul ainda é incerta. Gomes (1991) sugere que $M$. sorex seja sinonímia de $M$. brevicaudis (Olfers, 1818) e considera o segundo como nome válido para o estado. Dessa maneira, das três espécies deste gênero mencionadas por Rossi et al. (2006) como ocorrentes no Rio Grande do Sul, duas estão incluídas no grupo dimidiata (sensu Gomes 1991): M. dimidiata e $M$. brevicaudis (= $M$. sorex). A terceira espécie com ocorrência no estado, Monodelphis iheringi, está incluída no grupo americana (sensu Gomes 1991) e se diferencia morfologicamente das outras duas espécies pela presença de listas escuras na porção dorsal.

Métodos alternativos de identificação, de preferência nãoinvasivos, podem contribuir para a confirmação da ocorrência das espécies ou na identificação desses animais como presas de outros vertebrados. Um dos métodos não invasivos na identificação de mamíferos consiste da análise da morfologia interna dos pelos (Brunner \& Coman 1974, Teerink 1991, Fernández \& Rossi 1998, Quadros \& Monteiro-Filho 2006a). Quadros (2002) demonstrou a aplicação deste método na identificação de espécies de presas consumidas por predadores silvestres, analisando pelos encontrados em fezes. Vieira \& Port (2007) e Abreu et al. (2010) também se valeram deste método para determinar a espécie de predador através das amostras fecais dos mesmos e assim comparar a dieta de dois canídeos simpátricos no Rio Grande do Sul. Diversos autores já desenvolveram chaves de identificação de espécies baseadas exclusivamente em características tricológicas (Oli 1993, Perrin \& Campbel 1979, Wallis 1993, Mathiak 1938, Ingberman \& MonteiroFilho 2006).

O método de identificação de espécies de marsupiais utilizando a anatomia dos pelos pode contribuir para aumentar o conhecimento científico sobre esse grupo no sul do Brasil. O objetivo do presente estudo foi identificar padrões microscópicos da cutícula e medula de pelos-guarda de marsupiais com distribuição no Rio Grande do Sul, bem como propor uma chave dicotômica baseada nestes caracteres. Especificamente para o gênero Monodelphis, também objetivamos caracterizar possíveis morfotipos definidos por padrões de pelos distintos e apresentar a distribuição desses morfotipos no estado do Rio Grande do Sul.

\section{Material e Métodos}

Coletamos pelos de marsupiais a partir de animais taxidermizados e/ou conservados em meio líquido, depositados nas coleções das seguintes instituições brasileiras: Fundação Zoobotânica do RS, Laboratório de Ecologia de Mamíferos - UNISINOS, Museu de Ciências Naturais - ULBRA, Museu de Zoologia da Universidade de Brasília, Museu de Zoologia da Universidade Estadual de Campinas “Adão José Cardoso" (ZUEC), Parque Jardim Zoológico de Sapucaia do Sul - RS. Também obtivemos amostras de pelos diretamente de animais vivos, capturados durante o desenvolvimento de projetos de pesquisa realizados por nossos grupos. A lista completa dos espécimes utilizados para a remoção de pelos encontra-se no Anexo I.

Nas análises dos padrões tricológicos, utilizamos apenas pelosguarda, preferencialmente primários, de haste reta, não ondulada (Quadros 2002), coletados da região dorsal, na linha média anterior. Esses pelos se caracterizam por apresentar haste (porção do pelo mais próxima ao bulbo) e escudo (porção mais distal) bastante evidentes, o que facilita a identificação. Preparamos as lâminas pelo método sugerido por Quadros \& Monteiro-Filho (2006a), brevemente descrito a seguir. Para a identificação do padrão da cutícula (camada mais externa do pelo) pressionamos levemente o pelo em uma fina camada de esmalte de unha incolor e, após deixar para secar, retiramos o pelo com as mãos. Para identificação do padrão de medula (camada mais interna) o mesmo pelo foi posto em água oxigenada para descoloração. Utilizamos pó descolorante junto com a água oxigenada para acelerar o clareamento do pelo. Após o clareamento, lavamos o pelo e preparamos lâminas permanentes com entelan. Especificamente em relação à Didelphis, nós não fizemos cortes nos pelos, conforme sugeriram Quadros \& Monteiro-Filho (2006a). Essa prática foi sugerida pelos autores para aprimorar o clareamento dos pelos. Contudo, visto que não encontramos dificuldade em reconhecer os padrões mesmo sem utilizar esta prática, optamos por não realizála. Utilizamos a nomenclatura sugerida por Quadros \& MonteiroFilho (2006b) para identificar os padrões dos pelos-guarda. Todos os espécimes do gênero Monodelphis analisados tinham coloração relativamente uniforme (sem listras) e foram considerados como integrantes do grupo dimidiata, seguindo Gomes (1991). 
As lâminas foram analisadas em microscópio óptico, com aumento de 400x. Para obtenção das fotos, utilizamos máquina fotográfica digital acoplada à lente ocular do microscópio.

Analisamos um total de 131 espécimes de marsupiais, dos quais 80 foram agrupados em dez espécies: Caluromys lanatus $(\mathrm{n}=2$ espécimes), Chironectes minimus $(\mathrm{n}=7)$, Cryptonanus guahybae $(\mathrm{n}=2)$, Didelphis albiventris $(\mathrm{n}=21)$, Didelphis aurita $(\mathrm{n}=3)$, Gracilinanus agilis $(\mathrm{n}=4)$, Gracilinanus microtarsus $(\mathrm{n}=8)$, Lutreolina crassicaudata $(\mathrm{n}=14)$, Micoureus paraguayanus $(\mathrm{n}=4)$ e Philander frenatus $(\mathrm{n}=14)$. Os espécimes restantes foram classificados, a priori, somente em nível de gênero; 44 do gênero Monodelphis e sete do gênero Gracilinanus. Todos os espécimes identificados a nível de gênero foram capturados no Rio grande do Sul. A taxonomia de Didelphidae seguiu Gardner (2005), exceto para o gênero Cryptonanus, para o qual utilizamos Voss et al. (2005).

\section{Resultados}

As análises dos pelos indicaram combinações distintas para os padrões de cutícula e medula da maioria das espécies estudadas (Tabela 1, Figura 1, Figura 2), exceto para as espécies do gênero Gracilinanus analisadas (G. agilis e G. microtarsus) que apresentaram padrões similares. Nas outras espécies, encontramos diferenças na morfologia dos pelos. Não verificamos diferenças consistentes entre pelos de espécimes vivos, taxidermizados ou conservados em meio líquido.

A maioria das espécies que analisamos apresentou apenas uma combinação de padrão de cutícula e padrão de medula. A única exceção ocorreu em Didelphis albiventris. Nesta espécie encontramos dois padrões distintos na cutícula. Dos 21 espécimes analisados, seis apresentaram padrão de cutícula ondeada irregular (Figura 2c), enquanto todos os demais apresentaram cutícula folidácea larga.

Table 1. Patterns of hair medulla and cuticle of the didelphid marsupials studied. Classification and names of the observed patterns follow Quadros \& MonteiroFilho (2006b).

Tabela 1. Padrões medulares e cuticulares dos marsupiais didelfídeos estudados. Classificação e nomenclatura dos padrões de acordo com Quadros e MonteiroFilho (2006b).

\begin{tabular}{|c|c|c|c|c|c|c|c|c|c|c|c|c|c|}
\hline \multirow{2}{*}{$\begin{array}{c}\text { Características } \\
\text { dos pelos }\end{array}$} & \multicolumn{13}{|c|}{ Táxon } \\
\hline & Cla & Cmi & Cgu & Dal & Dau & Gag & Gmi & Lcr & Мpa & Mo1 & Mo2 & Mo3 & Pfr \\
\hline \multicolumn{14}{|l|}{ Medula } \\
\hline \multicolumn{14}{|l|}{ Unisseriada } \\
\hline \multicolumn{14}{|l|}{ Escalariforme } \\
\hline $1 / 2$ & $\mathrm{X}$ & - & - & - & - & $\mathrm{X}$ & $\mathrm{X}$ & - & $\mathrm{X}$ & $X$ & - & - & - \\
\hline $3 / 5$ & - & - & - & - & - & - & - & - & - & - & - & $\mathrm{X}$ & - \\
\hline \multicolumn{14}{|l|}{ Literácea } \\
\hline $1 / 2$ & - & - & $X$ & - & - & - & - & - & - & - & - & - & $\mathrm{X}$ \\
\hline $3 / 5$ & - & - & - & - & - & - & - & - & - & - & $\mathrm{X}$ & - & - \\
\hline \multicolumn{14}{|l|}{ Multisseriada } \\
\hline Reticulada & - & $\mathrm{X}$ & - & - & - & - & - & - & - & - & - & - & - \\
\hline \multicolumn{14}{|l|}{ Crivada } \\
\hline Simples & - & - & - & - & $\mathrm{X}$ & - & - & $X$ & - & - & - & - & - \\
\hline Trilobada & - & - & - & $\mathrm{X}$ & - & - & - & - & - & - & - & - & - \\
\hline \multicolumn{14}{|l|}{ Cutícula } \\
\hline \multicolumn{14}{|l|}{ Imbricada } \\
\hline \multicolumn{14}{|l|}{ Folidácea } \\
\hline Estreita & $\mathrm{X}$ & - & - & - & - & - & - & $X$ & - & - & - & - & - \\
\hline Intermediária & - & - & - & - & - & - & - & - & - & - & - & - & $\mathrm{X}$ \\
\hline Larga & - & - & - & $\mathrm{X}$ & $\mathrm{X}$ & - & - & - & - & - & - & - & - \\
\hline Conoidal & & & & & & - & - & - & - & - & - & - & - \\
\hline Simétrica & - & - & - & - & - & $\mathrm{X}$ & $\mathrm{X}$ & - & - & - & - & - & - \\
\hline Assimétrica & - & - & - & - & - & - & - & - & $\mathrm{X}$ & - & - & - & - \\
\hline Pavimentosa & - & - & - & - & - & - & - & - & - & - & - & - & - \\
\hline Ondeada & - & - & - & - & - & - & - & - & - & - & - & - & - \\
\hline Oblíqua & - & $\mathrm{X}$ & - & - & - & - & - & - & - & - & - & - & - \\
\hline Irregular & - & - & - & $\mathrm{X}$ & $\mathrm{X}$ & $\mathrm{X}$ & - & - & - & - & - & - & - \\
\hline Losângica & & & & & & - & - & - & - & - & - & - & - \\
\hline c/ eixo central & - & - & $\mathrm{X}$ & - & - & - & - & - & - & - & - & - & - \\
\hline s/ eixo central & - & - & - & - & - & - & - & - & - & $\mathrm{X}$ & $\mathrm{X}$ & $\mathrm{X}$ & - \\
\hline
\end{tabular}

Codes: $1 / 2=$ the medullar cells occupy half of the hair thickness; $3 / 5=$ the medullar cells occupy three fifths of the hair thickness. Cla $=$ Caluromys lanatus, $\mathrm{Cmi}=$ Chironectes minimus, $\mathrm{Cgu}=$ Cryptonanus guahybae, $\mathrm{Dal}=$ Didelphis albiventris, $\mathrm{Dau}=$ Didelphis aurita, Gag $=$ Gracilinanus agilis, $\mathrm{Gmi}=$ Gracilinanus microtarsus, $\mathrm{Lcr}=$ Lutreolina crassicaudata, $\mathrm{Mpa}=$ Micoureus paraguayanus, Mo1 = Monodelphis gr. dimidiata morphotype 1 , Mo2 = Monodelphis gr. dimidiata morphotype 2, Mo3 = Monodelphis gr. dimidiata morphotype 3, Pfr = Philander frenatus .

Códigos: $1 / 2$ = as células da medula ocupam metade da espessura do pelo; $3 / 5=$ células da medula ocupam três quintos da espessura do pelo. $\mathrm{Cla}=$ Caluromys lanatus, $\mathrm{Cmi}=$ Chironectes minimus, $\mathrm{Cgu}=$ Cryptonanus guahybae, Dal = Didelphis albiventris, Dau $=$ Didelphis aurita, $\mathrm{Gag}=$ Gra cilinanus agilis, Gmi = Gracilinanus microtarsus, Lcr = Lutreolina crassicaudata, Mpa = Micoureus paraguayanus, Mo1 = Monodelphis gr. dimidiata morfotipo 1, Mo2 = Monodelphis gr. dimidiata morfotipo 2, Mo3 = Monodelphis gr. dimidiata morfotipo 3, Pfr = Philander frenatus . 

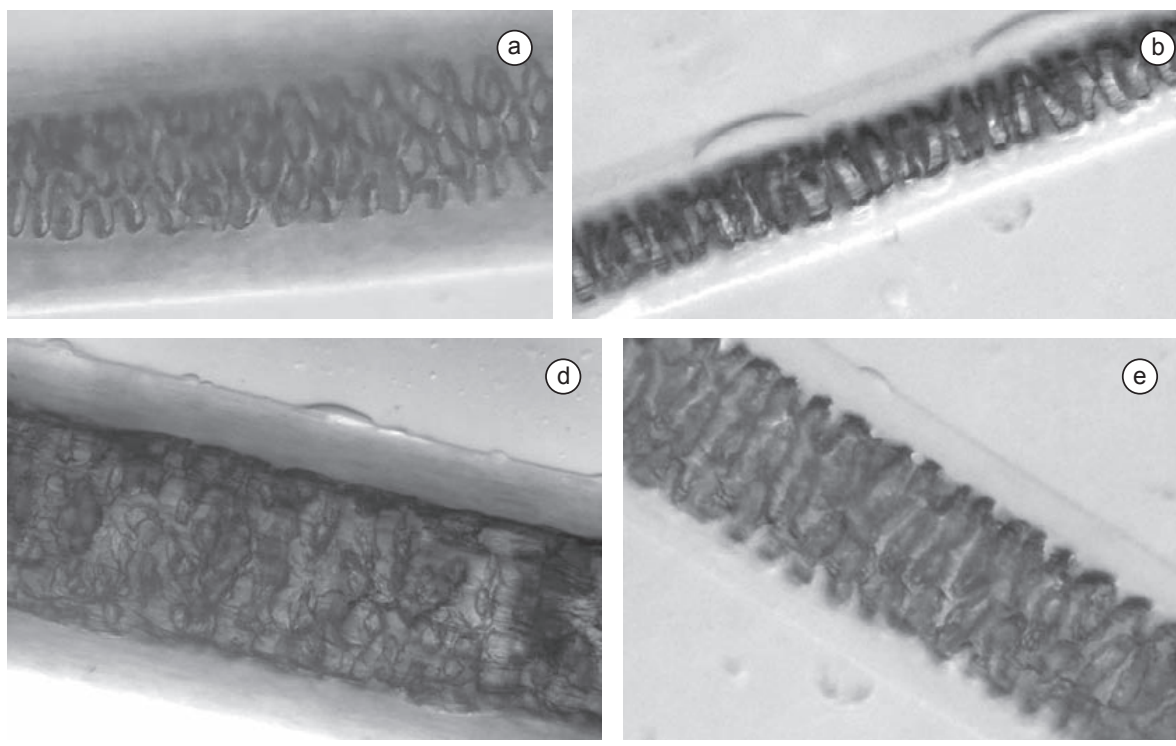

(e)
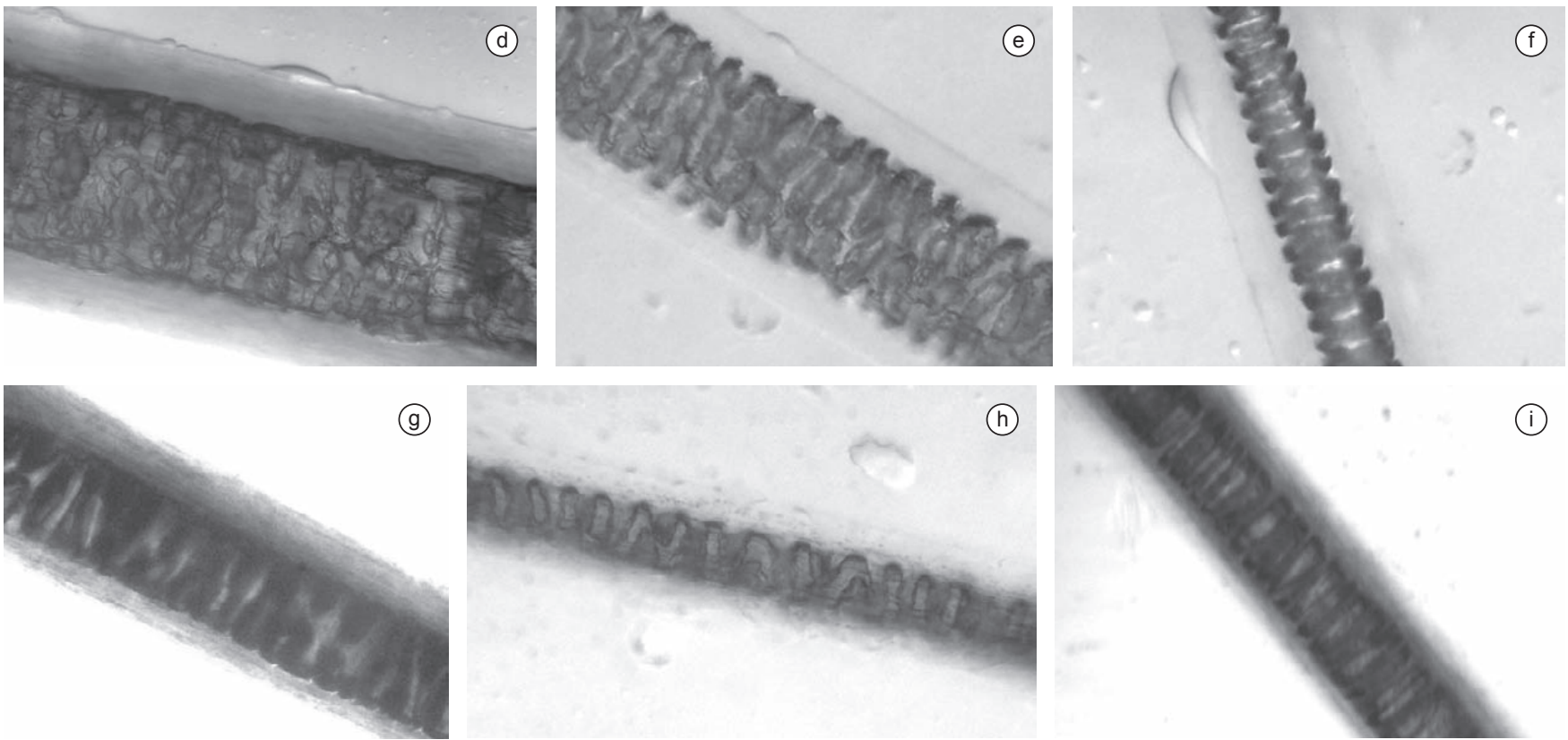

Figura 1.Padrões medulares na porção medial do escudo dos pelos dos marsupiais estudados (nomenclatura segundo Quadros \& Monteiro-Filho (2006b)): a) medula multisseriada reticulada (Chironectes minimus); b) medula unisseriada literácea, com células ocupando metade da espessura do pêlo (Cryptonanus guahybae); c) medula multisseriada crivada dividida em três lobos (Didelphis albiventris); d) medula multisseriada crivada simples (Didelphis aurita); e) medula multisseriada crivada simples, podendo lembrar listrada (Lutreolina crassicaudata); f) medula unisseriada escalariforme ocupando metade da espessura do pêlo (Caluromys lanatus, Gracilinanus agilis, Gracilinanus microtarsus, Micoureus paraguayanus e Monodelphis gr. dimidiata morfotipo 1); g) medula unisseriada literácea, com células ocupando três quintos da espessura do pêlo (Monodelphis gr. dimidiata morfotipo 2); h) medula unisseriada literácea (Philander frenatus); i) medula unisseriada escalariforme, com células ocupando três quintos da espessura do pêlo (Monodelphis gr. dimidiata morfotipo 3).

Figure 1. Medullar patterns on the medial portion of the shields of the marsupial hairs studied (for names see Quadros \& Monteiro-Filho (2006b)): a) multicelular reticulated medulla (Chironectes minimus); b) unicelular irregular medulla, with cells occupying half of the hair thickness (Cryptonanus guahybae); c) multicellular reticulated ('réticulée') medulla divided in three lobes (Didelphis albiventris); d) simple multicellular "réticulée" medulla (Didelphis aurita); e) simple multicellular reticulated (réticulée) medulla, sometimes looks like isolated (Lutreolina crassicaudata); f) unicellular ladder medulla, with cells occupying half of the hair thickness (Caluromys lanatus, Gracilinanus agilis, Gracilinanus microtarsus, Micoureus paraguayanus and Monodelphis gr. dimidiata morphotype 1); g) unicellular irregular medulla, with cells occupying three fifths of the hair thickness (Monodelphis gr. dimidiata morphotype 2); h) unicellular irregular medulla (Philader frenatus); i) unicellular ladder medulla, with cells occupying three fifths of the hair thickness (Monodelphis gr. dimidiata morphotype 3).

O padrão de cutícula ondeada irregular também foi registrado nos indivíduos de Didelphis aurita analisados. Contudo, as duas espécies se distinguiram pelo fato de que $D$. albiventris possui medula com três lobos na porção distal (Figura 1c), enquanto D. aurita apresenta somente um lobo. O terceiro lobo medular de D. albiventris aparece, em corte transversal, sobreposto às células da medula, dando a impressão de haver uma segunda camada de células sobre a medula. No microscópio óptico, este lobo apareceu sempre de modo desfocado.

Os espécimes de Micoureus paraguayanus apresentaram um padrão de cutícula e medula muito similar às duas espécies do gênero Gracilinanus. Eles se diferenciam pelo fato de que, em Micoureus paraguayanus, os dois lados do cone das escamas possuem alturas diferentes, padrão denominado de conoidal assimétrico (Figura 2f). Por outro lado, em Gracilinanus, as alturas são iguais, padrão reconhecido como conoidal simétrico (Figura 2d). Além disso, os exemplares de Gracilinanus apresentaram diferenças quando comparados a Micoureus paraguayanus na porção final da haste, na região de transição entre a haste e o escudo. Nesse local, Gracilinanus apresenta padrão de cutícula losângica (Figura 2g), enquanto que em Micoureus paraguayanus o padrão cuticular é ondeado (Figura 2c).

Em Monodelphis gr. dimidiata, todos os indivíduos apresentaram cutícula pavimentosa losângica estreita (Figura 2g). Entretanto, registramos a ocorrência de três padrões de medula. Dos 44 espécimes analisados, 12 indivíduos apresentaram medula unisseriada escalariforme, com células ocupando metade da espessura total do pelo (Figura 1f). Outros 16 apresentaram medula unisseriada literácea, com células ocupando metade ou mais do tamanho total do pelo (Figura 1g). Por fim, 16 indivíduos apresentaram medula unisseriada escalariforme, com células ocupando três quintos da espessura total do pelo (Figura 1i), o que permitiu classificar os espécimes como Monodelphis gr. dimidiata: morfotipo 1, com medula unisseriada escalariforme; morfotipo 2, com medula unisseriada literácea e 

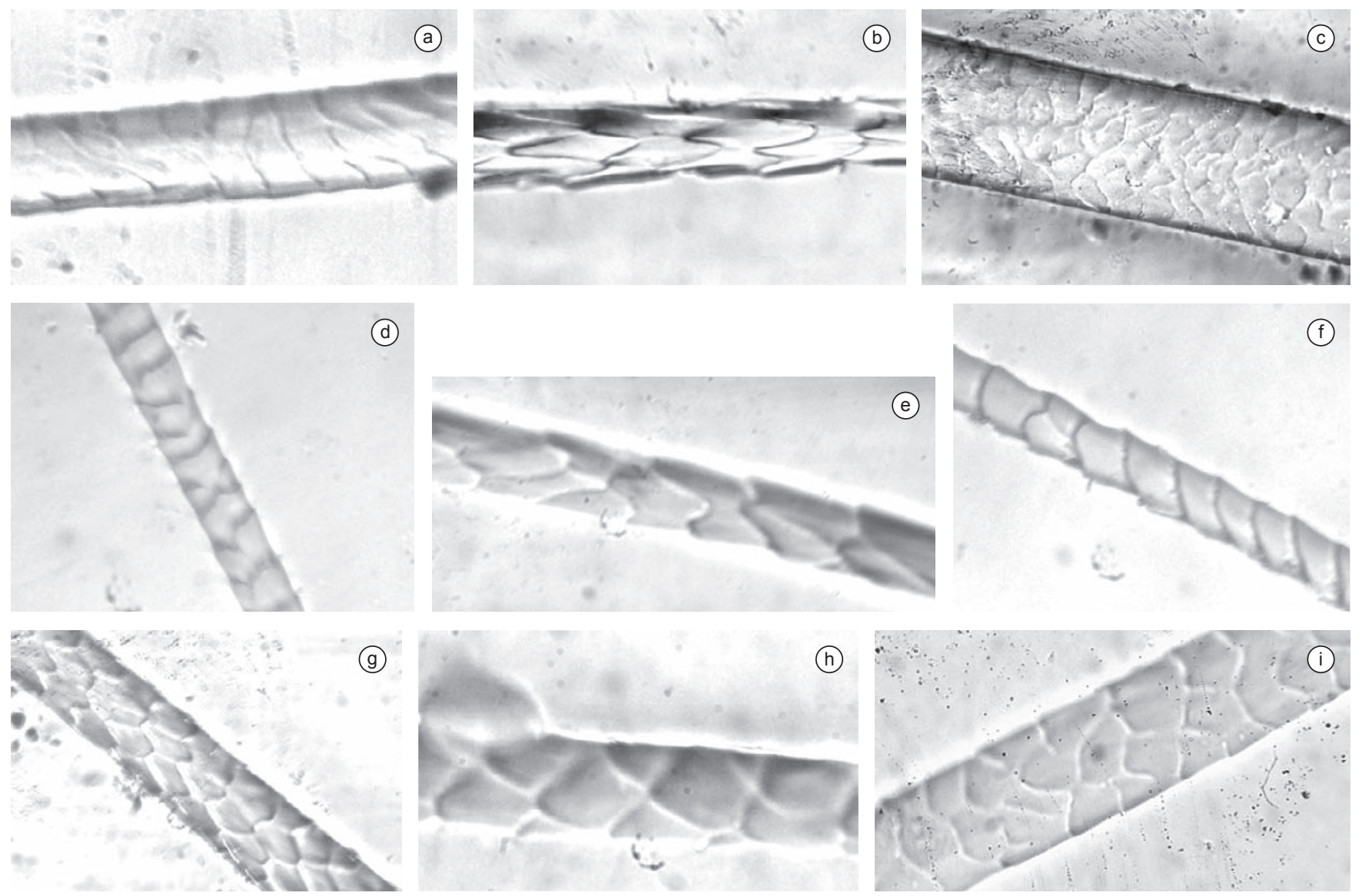

Figura 2. Padrões cuticulares na porção medial da haste dos marsupiais estudados (nomenclatura segundo Quadros \& Monteiro-Filho (2006b)): a) cutícula pavimentosa ondeada oblíqua simples (Chironectes minimus); b) cutícula pavimentosa losângica estreita, com eixo central aparente (Cryptonanus guahybae); c) cutícula pavimentosa ondeada irregular (Didelphis albiventris e Didelphis aurita); d) cutícula imbricada conoidal com ápice do cone de tamanhos iguais entre os lados (Gracilinanus agilis e Gracilinanus microtarsus); e) cutícula imbricada folidácea estreita (Caluromys lanatus e Lutreolina crassicaudata); f) cutícula imbricada conoidal com ápice do cone de tamanhos desiguais entre os lados (Micoureus paraguayanus); g) cutícula pavimentosa losângica estreita, sem eixo central aparente (Monodelphis gr. dimidiata); h) cutícula imbricada folidácea intermediária (Philander frenatus); i) cutícula imbricada folidácea larga (Didelphis albiventris e Caluromys lanatus).

Figure 2. Cuticle patterns on the medial portion of the shaft of the marsupials studied (for names see Quadros \& Monteiro-Filho (2006b)): a) simple oblique wave cuticle (Chironectes minimus); b) narrow non-imbricated diamond petal cuticle, with an apparent central axis (Cryptonanus guahybae); c) irregular non-imbricated waved cuticle (Didelphis albiventris and Didelphis aurita); d) symmetric imbricated conoidal cuticle (Gracilinanus agilis and Gracilinanus microtarsus); e) narrow imbricated petal cuticle (Caluromys lanatus and Lutreolina crassicaudata); f) asymmetric imbricated conoidal cuticle (Micoureus paraguayanus); g) narrow non-imbricated diamond petal cuticle, without apparent central axis (Monodelphis gr. Dimidiate); h) intermediate imbricated petal cuticle (Philander frenatus); i) wide imbricated petal cuticle (Didelphis albiventris and Caluromys lanatus).

morfotipo 3, com medula unisseriada escalariforme, porém com células ocupando três quintos da espessura total do pelo.

Os indivíduos de Cryptonanus guahybae analisados apresentaram um padrão de cutícula muito similar ao de Monodelphis gr. dimidiata morfotipo 2. A diferença entre estas espécies foi observada a partir da presença de um eixo central na cutícula losângica de C. guahybae, que era ausente em $M$. gr. dimidiata (Figura 2g). Em C. guahybae, foi possível verificar que as escamas de forma losângica se encaixam perfeitamente, com uma fileira de losangos centrais, na maior parte da extensão do pelo. O mesmo não foi visualizado em $M$. gr. dimidiata. Algumas características morfológicas macroscópicas também ajudaram na diferenciação. Em $M$. gr. dimidiata os pelos com padrão unisseriado literáceo eram mais espessos, mais escuros e mais curtos do que os pelos de C. guahybae. Também verificamos que, mesmo após o clareamento com água oxigenada, os pelos de $C$. guahybae apresentaram coloração bem mais clara, enquanto todos os indivíduos de $M$. gr. dimidiata possuíam uma coloração mais escura.

No gênero Monodelphis, verificamos a presença de dois padrões de medula unisseriada, escalariforme (Figuras 1f e 1i) e literáceo (Figura 1g). Separamos os padrões de medula unisseriada escalariforme em dois tipos, o primeiro com células que ocupam metade da espessura total do pelo (Figura 1f), ou menos, e o segundo com células que ocupam três quintos da espessura total do pelo, ou mais (Figura 1i). Registramos os locais de ocorrência, no Rio Grande do Sul, de cada um dos três padrões de medula que encontramos (Figura 3). Todos os exemplares desse gênero, utilizados no estudo, foram capturados no centro, norte e nordeste do Rio Grande do Sul. Dos três padrões que encontramos para a medula, o padrão de medula unisseriada escalariforme, com células ocupando metade da espessura do pelo (morfotipo 1), foi aquele que apresentou maior distribuição. O padrão de medula literácea (morfotipo 2) foi muito mais restrito. Este último foi registrado principalmente no centro do estado, onde houve a sobreposição dos três morfotipos.

Os padrões anatômicos de cutícula e medula encontrados nos pelos das espécies analisadas nos permitiram organizar uma chave para identificação de espécies de marsupiais com ocorrência no Rio Grande do Sul (Anexo I). 


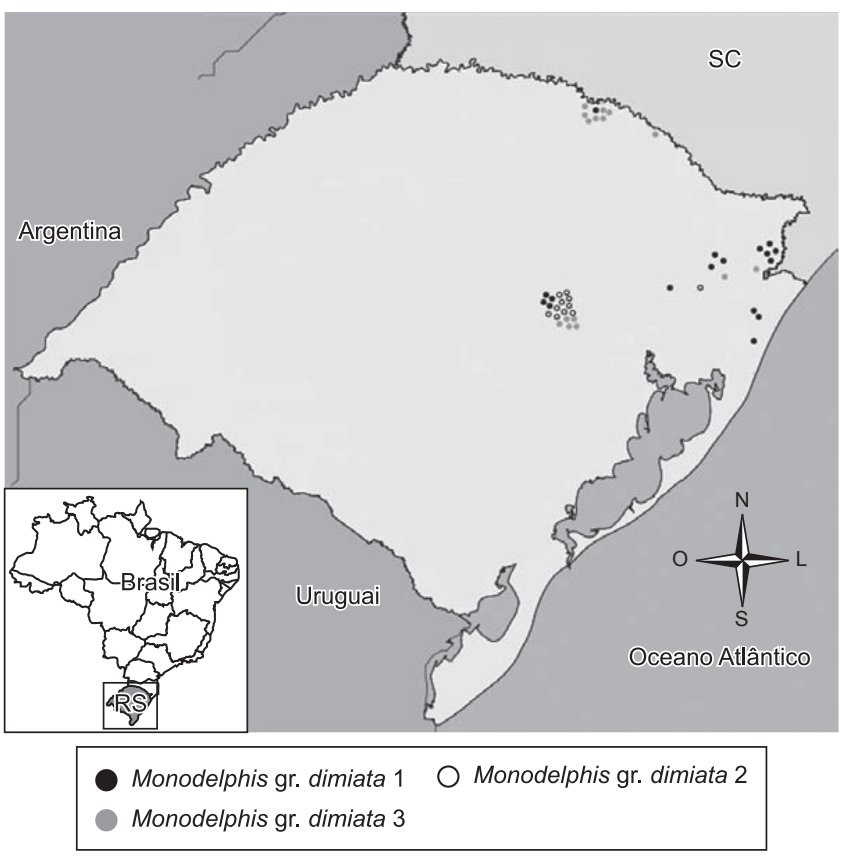

Figura 3. Distribuição, no estado do Rio Grande do Sul, dos morfotipos associados aos distintos padrões microscópicos de pêlos-guarda dos espécimes do gênero Monodelphis analisados no estudo (apenas espécimes com localização confirmada). Cada ponto refere-se a um indivíduo. Todos os indivíduos apresentaram cutícula com padrão pavimentoso losângico estreito (Figura 2g).

Figure 3. Geographic distribution of the Monodelphis morphotypes with distinct microscopic patterns of their guard-hairs in Rio Grande do Sul state. Only specimens with confirmed localization were considered. Each point represents an individual. All the individuals presented narrow non-imbricated diamond petal cuticle (Figure 2g).

\section{Discussão}

Muito poucos estudos realizaram análise da micro-estrutura dos pelos de pequenos mamíferos brasileiros (Quadros \& Monteiro-Filho 1998, Quadros 2002, Martin et al. 2009). Das espécies estudadas por Quadros (2002), apenas para o padrão cuticular de D. albiventris encontramos resultados diferentes dessa autora. Nesse estudo, há a indicação apenas do padrão ondeado para essa espécie (Quadros 2002), enquanto nós encontramos também um padrão folidáceo. Contudo, esse padrão folidáceo já havia sido registrado anteriormente para D. albiventris (Quadros \& Monteiro-Filho 1998). Em outro estudo, que investigou os padrões de cutícula e medula de marsupiais e roedores de São Paulo (Martin et al. 2009), houve o registro de um padrão de cutícula desse marsupial similar ao descrito por Quadros (2002). Por outro lado, Martin et al. (2009) obtiveram resultados diferentes para a cutícula de Gracilinanus microtarsus em relação aos resultados obtidos por Quadros (2002) e os que obtivemos no presente estudo. Embora os estudos nesta área ainda sejam muito escassos e ainda haja muitas questões a serem respondidas, podemos sugerir que essas duas espécies podem apresentar certa variação longitudinal no padrão de cutícula de seus pelos. No caso de D. albiventris, alguns dos espécimes que analisamos no presente estudo são provenientes do estado de São Paulo, sendo esses os espécimes que apresentaram maior proporção de padrão ondeado. Somente dois dos espécimes provenientes do Rio Grande do Sul apresentaram este padrão de cutícula. Essa mesma variação não foi observada na medula, uma vez que os três estudos aqui citados encontraram os mesmos resultados no padrão de medula para essas duas espécies de marsupiais.

A maioria dos marsupiais que analisamos no presente estudo tem padrão de medula distinto dos padrões previamente identificados para roedores que ocorrem no RS, como Delomys dorsalis (Hensel, 1873), Oligoryzomys nigripes (Olfers, 1818), Oryzomys sp., Oxymycterus sp., Akodon montensis (Thomas, 1913), Necromys lasiurus (Lund, 1840) e Bibimys labiosus (Winge, 1887) (Quadros 2002, Martin et al. 2009). A única exceção foi Kannabateomys amblyonyx (Wagner, 1845) (Rodentia, Echimyidae), cujo padrão identificado por Martin et al. (2009) é similar ao que encontramos para Didelphis aurita. Isso indica que a técnica que utilizamos permitiria, no mínimo, a diferenciação de pequenos mamíferos predados por grandes vertebrados (principalmente carnívoros) entre roedores e marsupiais, a partir de pelos coletados em fezes ou conteúdo estomacal. Kraus \& Rödel (2004) utilizaram esta técnica para identificar mamíferos predados por furão (Galictis cuja). Além disso, a técnica também pode ser utilizada para levantamentos faunísticos, através de armadilhas de pelos (e.g. Fasola et al. 2005, Pocock \& Jennings 2006) e identificação de ninhos utilizados por estes animais. Obviamente para uma melhor identificação seria preciso analisar e verificar quais os padrões microscópicos de pelos dos roedores que ocorrem no estado.

O padrão de medula trilobada que encontramos para $D$. albiventris também foi detectado por Müller (1989) através de corte transversal. Quadros (2002) registrou visualização deste aspecto sem a necessidade de corte transversal. Em nosso estudo, também registramos a presença destes lobos sem a necessidade de corte transversal. Devido à presença destes lobos, os pelos apareceram em duas perspectivas, estando uma delas sempre desfocada.

Estudos de dieta de vertebrados de pequeno e médio porte que atuam como predadores de mamíferos podem ser incompletos, caso se baseiem somente no registro de partes duras remanescentes nas fezes. Analisando a dieta de M. dimidiata, Busch \& Kravetz (1991) encontraram pelos de roedores de diferentes espécies e classes de tamanho nos estômagos analisados. Restos de ossos, entretanto, foram encontrados apenas para os roedores maiores. Já Vieira \& Palma (1996), observaram que, ao menos em cativeiro, Thylamys velutinus (Wagner, 1842) pode se alimentar de pequenos roedores. No entanto, a investigação da dieta desse marsupial por meio de análise do conteúdo fecal não confirmou a presença de nenhum mamífero com base na identificação por ossos e outras partes duras remanescentes (Vieira \& Palma 1996). Desta maneira, sugerimos que especialmente para avaliação da dieta de pequenos predadores, o uso dos pelos para identificar a presença de mamíferos de pequeno porte possa ser tão ou mais relevante do que o uso de ossos.

No presente estudo, para dois espécimes de Monodelphis, analisamos os padrões dos pelos do dorso e do ventre e não verificamos diferenças marcantes entre essas regiões do corpo. Isso indica que diferenças na localização original dos pelos no corpo do animal não interferem de forma acentuada na utilização dos mesmos para identificação da espécie. Por uma questão de padronização, entretanto, optamos por coletar os pelos de todos os demais espécimes apenas na região dorsal. Fernández \& Rossi (1998), em uma análise igualmente baseada em padrões de medula e cutícula, também detectaram similaridade nos pelos-guarda de diferentes regiões do corpo de pequenos mamíferos (roedores e um marsupial (Thylamys (= Marmosa) pusillus [Desmarest, 1804]) da Argentina.

Os resultados que obtivemos permitiram separar Gracilinanus microtarsus e Cryptonanus guahybae pelo padrão da medula e da cutícula. Até pouco tempo, Cryptonanus guahybae era considerada uma sub-espécie do gênero Gracilinanus (Gracilinanus microtarsus guahybae; Voss et al. (2005)). O resultado que obtivemos no presente estudo sugerem a presença de diferenças 
morfológicas marcantes no nível tricológico entre essas duas espécies. Contudo, não encontramos diferenças tão marcantes entre as duas espécies do gênero Gracilinanus.

O fato de termos encontrado, entre os espécimes do gênero Monodelphis, três padrões de medula levanta duas hipóteses não excludentes. A primeira é a ocorrência de três espécies válidas para este grupo (dimidiata) no estado. A segunda hipótese é que exista uma variação intra-específica entre os representantes do gênero. No Rio Grande do Sul, há a ocorrência confirmada de pelo menos três espécies do gênero, sendo $M$. iheringi pertencente ao grupo americana (sensu Gomes 1991) (Emmons \& Feer 1990, Brown 2004, Rossi et al. 2006). No entanto, não existe consenso entre autores para a nomenclatura das espécies do grupo dimidiata. Gomes (1991) e Pine et al. (1985) incluem M. henseli (Thomas, 1888) como sinônimo de M. sorex (Hensel, 1872), uma espécie que é citada por Emmons $\&$ Feer (1990) e Rossi et al. (2006) com registro no Estado. Baseado em uma revisão de todo o gênero Monodelphis, entretanto, Gomes (1991) cita M. brevicaudis (Olfers, 1818) como um nome válido para essa espécie. O padrão de cutícula losângica que encontramos para todos os indivíduos do gênero Monodelphis corrobora com o padrão anteriormente descrito por Quadros (2002) para Monodelphis sorex. Isso reforça a possibilidade de $M$. sorex ser, no Rio Grande do Sul, sinonímia de $M$. brevicaudis.

Não incluímos, em nosso estudo, nenhum indivíduo de $M$. iheringi nas análises, devido ao fato de não termos encontrado nas coleções científicas visitadas nenhum espécime que tenha sido capturado no estado. Quadros (2002) descreveu o padrão de cutícula dessa espécie como folidáceo estreito na haste, e o padrão da medula como literáceo com margens crenadas. Este padrão é similar ao que encontramos para Philander frenatus. Em seu estudo, a autora mencionou diferenças entre estas espécies nas margens da medula (ondeadas em $P$. frenatus e crenadas em $M$. iheringi) e as formações de literóides (conspícua em $P$. frenatus e inconspícua em $M$. iheringi). Contudo, visto que não dispomos de pelos de $M$. iheringi para análises, esta espécie não foi incluída na chave de identificação proposta neste estudo.

Dos três padrões de medula que identificamos para Monodelphis, dois são muito similares (os dois padrões de medula unisseriada escalariforme). Eles se diferenciam apenas pelo tamanho que as células ocupam em relação à espessura total do pelo. Esta pequena diferença pode ser apenas uma variação individual, de faixa etária ou estado nutricional, e desta forma ambas representariam a mesma espécie. O fato de estes dois padrões ocorrerem em espécimes simpátricos em algumas regiões (norte e centro do estado) reforça esta possibilidade. Entretanto, como alguns destes animais demonstraram pequenas porém consistentes diferenças morfológicas (como tamanho do corpo e coloração dos pelos), acreditamos que os padrões tricológicos observados possam estar associados a possíveis diferenças inter-específicas. Para um melhor esclarecimento, seriam necessárias análises de um maior número de espécimes, além de identificações aprimoradas dos espécimes do gênero analisados, através de medidas cranianas e/ou marcadores moleculares.

A análise dos padrões microscópicos de pelos de mamíferos é uma técnica não-invasiva que distingue, com sucesso, a maioria das espécies de marsupiais do Rio Grande do Sul. Essa técnica pode ser usada para auxiliar na identificação de pequenas presas consumidas por vertebrados e também para discriminar espécies com morfologia externa similar. Além disso, por meio da identificação de pelos obtidos com armadilhas de pelos, pode servir para a confirmação da ocorrência de espécies que não tenham sido capturadas com outros métodos em uma determinada área. Os resultados obtidos para o gênero Monodelphis levantam a possibilidade de que as espécies do grupo dimidiata já reconhecidas (M. brevicaudis e $M$. dimidiata) possam ocorrer com uma distribuição distinta no estado, estando uma presente principalmente na porção norte e a outra na porção leste do RS, com alguma sobreposição na região central.

\section{Agradecimentos}

Somos gratos pela concessão de material biológico para as análises tricológicas aos curadores das seguintes instituições: Coleção de Mamíferos do Museu de Ciências Naturais da ULBRA; Dra. Márcia Jardim, da Fundação Zoobotânica do Rio Grande do Sul; Dr. Jader Marinho-Filho, do Museu de Zoologia da Universidade de Brasília (UnB); e a Dr a A. Cecília Z. Amaral, do Museu de Zoologia “Adão José Cardoso"da Universidade Estadual de Campinas. Também agradecemos aos colegas e pesquisadores do Laboratório de Ecologia de Mamíferos da UNISINOS pela coleta de material, pelas discussões e sugestões e a dois revisores pelos comentários feitos a uma versão anterior desse artigo. Durante a execução deste estudo, EMV recebeu apoio financeiro do Conselho Nacional de Pesquisa e Desenvolvimento Tecnológico CNPq (Bolsa de Produtividade em Pesquisa, Processo no 300286/99-6) e MSLA recebeu bolsa de iniciação científica do CNPq (PIBIC, Processo nº 800479/1993-9).

\section{Referências Bibliográficas}

ABREU, M.S.L., WIELICZKO, A.R., MESQUITA, A. \& VIEIRA, E.M. 2010. Consumo de pequenos mamíferos por canídeos simpátricos do sul do Brasil: sobreposição de nichos e seleção de presas. Neotrop. Biol. Conserv. 5(1):16-23. http://dx.doi.org/10.4013/nbc.2010.51.03

BROWN, B.E. 2004. Atlas of new world marsupials. Fieldiana 30:308.

BRUNNER, H. \& COMAN, B.J. 1974. The identification of mammalian hair. Inkata Press, Melbourne.

BUSCH, M. \& KRAVETZ, F.O. 1991. Diet composition of Monodelphis dimidiata (Marsupialia, Didelphidae). Mammalia 55(4):619-621.

CÁCERES, N.C. 2002. Food habits and seed dispersal by the white-eared opossum, Didelphis albiventris, in southern Brazil. Stud. Neotrop. Fauna Env. 37(2):97-104.

CÁCERES, N.C \& MONTEIRO-FILHO, E.L.A. 2000. The common opossum, Didelphis aurita, as a seed disperser of several plants in southern Brazil. Cienc. Cult. 52(1):41-44.

CÁCERES, N.C., DITTRICH, V.A.O. \& MONTEIRO-FILHO, E.L.A. 1999. Fruit consumption, distance of seed dispersal and germination of solanaceous plants ingested by common opossum (Didelphis aurita) in southern Brazil. Rev. Ecol. (Terre Vie) 54(3):225-234.

CANEVARI, M. \& VACCARO, O. 2007. Guía de mamíferos del sur de América del Sur. Literature of Latin América, Buenos Aires.

EMMONS, L.H. \& FEER, F. 1990. Neotropical rainforest mammals: a field guide. The University of Chicago Press, Chicago.

FASOLA, L., BELLO, M. \& GUICHÓN, M.L. 2005. Uso de trampas de pelo y caracterización de los pelos de la andilla de vientre rojo Collosciurus erythraeus. Mastozool. Neotrop. 12(1):9-17.

FERNÁNDEZ, G.J. \& ROSSI, S.M. 1998. Medullar type and cuticular scale patterns of hairs of rodents and small marsupials from the monte scrubland (San Luis Province, Argentina). Mastozool. Neotrop. 5(2):109-116.

GARDNER, A. 2005. Order Didelphimorphia. In Mammal species of the world: a taxonomic and geographic reference (D.E. Wilson \& D.A.M. Reeder, eds.). 3nd ed. The Johns Hopkins University Press, Baltimore, Maryland, p.3-18.

GATTI, A., BIANCHI, R., ROSA, C.R.X. \& MENDES, S.L. 2006a. Diet of crab-eating fox, Cerdocyon thous (Carnivora, Canidae) in Paulo Cesar Vinha State Park, Espírito Santo State, Brazil. Mammalia 70(1-2):153155. http://dx.doi.org/10.1515/MAMM.2006.021

GATTI, A., BIANCHI, R., ROSA, C.R.X. \& MENDES, S.L. 2006b. Diet of two sympatric carnivores, Cerdocyon thous and Procyon cancrivorus, in a restinga area of Espirito Santo State, Brazil. J. Trop. Ecol. 22:227-230. http://dx.doi.org/10.1017/S0266467405002956 
GOMES, N.F. 1991. Revisão sistemática do gênero Monodelphis (Didelphidae: Marsupialia). Tese de Mestrado, Universidade de São Paulo, São Paulo.

GRIBEL, R. 1988. Visits of Caluromys lanatus (Didelphidae) to flowers of Pseudobombax tomentosum (Bombacaceae): a probable case of pollination by marsupials in central Brazil. Biotropica 20(4):344-347. http://dx.doi.org/10.2307/2388329

INGBERMAN, B. \& MONTEIRO-FILHO, E.L.A. 2006. Identificação microscópica dos pelos das espécies brasileiras de Alouatta Lacépède, 1799 (Primates, Atelidae, Alouattinae). Arq. Mus. Nac. Rio de Janeiro 61(1):61-71.

JÁCOMO, A.T.A., SILVEIRA, L. \& DINIZ-FILHO, J.A.F. 2004. Niche separation between the maned wolf (Chrysocyon brachyurus), the crabeating fox (Dusicyon thous) and the hoary fox (Dusicyon vetulus) in central Brazil. J. Zool. 262:99-106.

JANSA, S.A. \& VOSS, R.S. 2000. Phylogenetic studies on didelphid marsupials I: introduction and preliminary results from IRBP gene sequences. $J$. Mamm. Evol. 7(1):43-77. http://dx.doi.org/10.1023/A:1009465716811

JANSA, S.A. \& VOOS, R.S. 2005. Phylogenetic relationships of the marsupial genus Hyladelphys based on nuclear gene sequences and morphology. J. Mammal. 86(5):853-865. http://dx.doi.org/10.1644/15451542(2005)86[853:PROTMG]2.0.CO;2

JANSA, S. A., FORSMAN, J. F., VOSS, R.S. 2006. Different patterns of selection on the nuclear genes IRBP and DMP-1 affect the efficiency but not the outcome of phylogeny estimation for didelphid marsupials. Mol. Phylogenet. Evol. 38(2):363-380. PMid:16054401. http://dx.doi. org/10.1016/j.ympev.2005.06.007

KRAUS, C. \& RÖDEL, H.G. 2004. Where have all the cavies gone? Causes and consequences of predation by the minor grison on a wild cavy population. Oikos 105(3):489-500. http://dx.doi.org/10.1111/j.00301299.2004.12941.x

MARTIN, P.S., GHELER-COSTA, C. \& VERDADE, L.M. 2009. Microestruturas de pelos de pequenos mamíferos não-voadores: chave para identificação de espécies de agroecossistemas do estado de São Paulo, Brasil. Biota Neotrop. 9(1): http://www.biotaneotropica.org.br/ v9n1/pt/abstract?identification-key+bn01509012009 (ultimo acesso em 17/03/2011).

MATHIAK, H.A. 1938. A key to hairs of the mammals of southern Michigan. J. Wildl. Managem. 2(4):251-269. http://dx.doi.org/10.2307/3795673

MÜLLER, M.V.Y. 1989. Microestrutura de pêlos de mamíferos: métodos de análise e sua aplicação na identificação de algumas espécies do estado do Paraná, Brasil. Tese de Mestrado, Universidade Federal do Paraná, Curitiba.

OLI, M.K. 1993. A key for the identification of the hair of mammals of a snow leopard (Panthera uncia) habitat in Nepal. J. Zool. 231:71-93. http:// dx.doi.org/10.1111/j.1469-7998.1993.tb05354.x

PATTON, J.L.\& DA SILVA, M.N.F. 1997. Definition of species of pouched four eyed opossums (Didelphidae, Philander). J. Mammal. 78(1):90-102. http://dx.doi.org/10.2307/1382642

PEDÓ, E., TOMAZZONI, A.C., HARTZ, S.M. \& CHRISTOFF, A.U. 2006. Diet of crab-eating fox, Cerdocyon thous (Linnaeus) (Carnivora, Canidae), in a suburban area of southern Brazil. Rev. Bras. Zool. 23(3):637-641.

PERRIN, M.R. \& CAMPBELL, B.S. 1979. Key to the mammals of the Andries Vosloo Kudu Reserve (eastern Cape), based on their hair morphology, for use in predator scat analysis. S. Afr. J. Wildl. Res 10(1):1-14.
PINE, R.H., DALBY, P.L. \& MATSON, J.O. 1985. Ecology, postnatal development, morphometrics, and taxonomic status of the short-tailed opossum, Monodelphis dimidiata, an apparently semelparous annual marsupial. Ann. Carn. Mus. 54(6):195-231.

POCOCK, M.J.O. \& JENINNGS, N. 2006. Use of hair tubes to survey of shrews: new methods for identification and quantification of abundance. Mammal Rev. 36(4):299-308. http://dx.doi.org/10.1111/j.13652907.2006.00092.x

QUADROS, J. 2002. Identificação microscópica de pelos de mamíferos brasileiros e sua aplicação no estudo da dieta de carnívoros. Tese de Doutorado, Universidade Federal do Paraná, Curitiba.

QUADROS, J. \& MONTEIRO-FILHO, E.L.A. 1998. Effects of digestion, putrefaction, and taxidermy processes on Didelphis albiventris hair morphology. J. Zool. 244:331-334. http://dx.doi. org/10.1111/j.1469-7998.1998.tb00037.x

QUADROS, J. \& MONTEIRO-FILHO, E.L.A. 2006a. Coleta e preparo de pelos de mamíferos para identificação em microscopia óptica. Rev. Bras. Zool. 23(1):274-278. http://dx.doi.org/10.1590/S010181752006000100022

QUADROS, J. \& MONTEIRO-FILHO, E.L.A. 2006b. Revisão conceitual, padrões microestruturais e proposta nomenclatória para os pelos-guarda de mamíferos brasileiros. Rev. Bras. Zool. 23(1):279-292. http://dx.doi. org/10.1590/S0101-81752006000100023

ROSSI, R.V.; BIANCONI, G.V.; PEDRO, W.A. 2006. Ordem Didelphimorphia. In Mamíferos do Brasil (N.R. Reis, A.L. Peracchi, W.A. Pedro \& I.P. Lima, eds.). Universidade Estadual de Londrina, Londrina, p.27-66.

SARTI, P.T. 2009. Efeito de borda em pequenos mamíferos do sul do Brasil: variação entre áreas, relação com o uso do hábitat e estratificação vertical. Tese de Mestrado, Universidade do Vale do Rio dos Sinos, São Leopoldo.

TEERINK, B.J. 1991. Hair of West European mammals: atlas and identification. Cambridge University Press, Cambridge.

VIEIRA, E. M. \& IOB, G. 2003. Marsupiais. In Livro vermelho da fauna ameaçada de extinção no Rio Grande do Sul (C.S. FONTANA, G.A. BENCKE \& R.E. REIS eds). EDIPURS, Porto Alegre, p. 481-486.

VIEIRA, E.M. \& PALMA, A.R.T. 1996. Natural history of Thylamys velutinus (Marsupialia, Didelphidae) in central Brazil. Mammalia 60(3):481-484.

VIEIRA, E.M. \& PORT, D. 2007. Niche overlap and resource partitioning between two sympatric fox species in southern Brazil. J. Zool. 272(1):5763. http://dx.doi.org/10.1111/j.1469-7998.2006.00237.x

VIEIRA, M.F., CARVALHO-OKANO, R.M. \& SAZIMA, M. 1991. The common opossum, Didelphis marsupialis, as a pollinator of Mabea fistulifera (Euphorbiaceae). Cienc. Cult. 43(5):390-393.

VOSS, R.S. \& JANSA, S.A. 2003. Phylogenetic studies on didelphid marsupials II. Nonmolecular data and new IRBP sequences: Separate and combined analyses of didelphine relationships with denser taxon sampling. Bull. Amer. Mus. Nat. Hist. 276:82 pp.

VOSS, R.S., YENSEN, E., TARIFA, T. 2004. An introduction to Marmosops (Marsupialia: Didelphidae), with the description of a new species from Bolivia and notes on the taxonomy and distribution of other Bolivian congeners. Am. Mus. Novit. 3466:1-40. http://dx.doi.org/10.1206/00030082(2004)466\%3C0001:AITMMD\%3E2.0.CO;2

VOSS, R.S., LUNDE, D.P. \& JANSA, S.A. 2005. On the contents of Gracilinanus Gardner and Creighton, 1989, with the description of a previously unrecognized clade of small didelphid marsupials. Am. Mus. Novit. 3482:35 pp.

WALLIS, R.L. 1993. A key for the identification of guard hairs of some Ontario mammals. Can. J.Zool. 71(3):587-591. http://dx.doi.org/10.1139/ z93-080 
Anexo I. Lista de exemplares utilizados no presente estudo (92 espécimes)

Annex I. List of the specimens used in the present study (92 specimens).

Caluromys lanatus (Olfers 1818) $(\mathrm{n}=2)$

UnB: 400 (Brasília, DF). UNICAMP: 1520 (sem registro de procedência).

Chironectes minimus (Zimmermann 1780) $(\mathrm{n}=7)$

LEM: LEM-178 (Maquine, RS). MCNU: 255 (sem registro de procedência). UnB: 398 (Jataí, Goiás), 399 (Brasília, DF), 439 (Majé, RJ). UNICAMP: 2385 (Intervales, SP). ZOO: $\mathrm{Cm} \mathrm{s} / \mathrm{n}^{\circ}$ (sem registro de procedência).

Cryptonanus guahybae (Tate 1931) $(\mathrm{n}=2)$

LEM: LEM-197 (Candiota, RS), LEM-198 (Rio Grande, RS).

Didelphis albiventris (Lund 1840) $(\mathrm{n}=21)$

FZB: $D a \mathrm{~s} / \mathrm{n}^{\circ}$ (sem registro de procedência), 018 (Sapucaia do Sul, RS), 00171 (Cerrito, RS), 00338 (Porto Alegre, RS), 1702 (Porto Alegre, RS), FS-188 (Santa Maria, RS), FS-282 (São Leopoldo, RS), FS-300 (Triunfo, RS), FS-528 (Montenegro, RS), MCN-345 (sem registro de procedência), PG-001 (Pântano Grande, RS), PG-002 (Panambi, RS), PG-006/1 (Cabriuva, RS), PG-013 (Porto Alegre, RS). LEM: LEM-206 (São Leopoldo, RS). MCNU: 235 (Caraá, RS), 237 (Torres, RS), 533 (Capão da Canoa, RS). UNICAMP: 1213 (Mogi Guaçu, SP), 1412 (Campinas, SP), 1420 (Campinas, SP).

Didelphis aurita (Wied-Neuwied 1826) $(\mathrm{n}=3)$

FZB: $D a$ s $/ n^{\circ}$ (sem registro de procedência), MCNU-00421 (Torres, RS). MCNU: 070 (ES).

Gracilinanus agilis (Burmeister 1854) $(\mathrm{n}=4)$

LEM: MZUN-252 (Capinzal, SC). UnB: 539 (Brasília, DF), 1854 (Jatobá, BA), 1862 (Manso, MT).

Gracilinanus microtarsus (Wagner 1842) $(\mathrm{n}=8)$

ARA*: ARA-39, ARA-040. FLONA*: PT-204. UNICAMP: 1513 (Américo Brasiliensis, SP), 1963 (Intervales, SP), 2196 (Campinas, SP). UnB: 735 (Teresópolis, RJ), 1277 (Linhares, ES).

Gracilinanus sp. (Gardner \& Creighton 1989) $(\mathrm{n}=7)$

FZB: RFB-0073 (Barra Grande, RS), RFB-0206 (Barra Grande, RS), RFB-0411 (Barra Grande, RS), RFB-0662 (Barra Grande, RS). LEM: 431 (sem registro de procedência), LEM-215 (Barra Grande, RS). MCNU: JR345 (sem registro de procedência).

Lutreolina crassicaudata (Desmarest 1804) $(\mathrm{n}=14)$

FZB: 00227 (Porto Alegre, RS), 1055 (Reserva Ecológica do Taim, Rio Grande, RS), FZB-037 (BR-290, RS), MCN-426 (Porto Alegre, RS). LEM: LEM-190 (Mostardas, RS). MCNU: 025 (Quintão, RS), 241. UnB: 397 (Jataí, GO), 1707 (Parque Nacional de Emas, GO). UNICAMP: 1471 (Campinas, SP), 1521 (Piracicaba, SP), 1794 (Campinas, SP), 1855 (Campinas, SP), 2299 (sem registro de procedência).

Micoureus paraguayanus (Tate 1931) $(\mathrm{n}=4)$

FZB: $M d$ s/no (Osório, RS). MCNU: 216 (Gravataí, RS). UnB: 422 (Magé, RJ), 419 (Magé, RJ).
Monodelphis gr. dimidiata (Gomes 1991) $(\mathrm{n}=44)$

FLONA*: GI-1060, GI-1127. FZB: MCN-430 (RS), MCN2882 (Nova Petrópolis, RS), RFB-0306 (Barra Grande, RS), Md s/no (Osório, RS). MCNU: 134 (Maquine, RS), 135 (Venâncio Aires, RS), 136 (Venâncio Aires, RS), 154 (Venâncio Aires, RS), 155 (Venâncio Aires, RS), 156 (Venâncio Aires, RS), 157 (Venâncio Aires, RS), 158 (Venâncio Aires, RS), 159 (Venâncio Aires, RS), 160 (Venâncio Aires, RS), 161 (Venâncio Aires, RS), 162 (Venâncio Aires, RS), 163 (Venâncio Aires, RS), 164 (Canela, RS), 166 (Venâncio Aires, RS), 168 (Venâncio Aires, RS), 169 (Venâncio Aires, RS), 171 (Venâncio Aires, RS), 173 (Venâncio Aires, RS), 202 (Maquiné, RS), 232 (Venâncio Aires, RS), 233 (Venâncio Aires, RS). LEM: 223 (Cambará do Sul, RS), 1000 (Maximiliano de Almeida, RS), 1377 (Maximiliano de Almeida, RS), 1736 (Maximiliano de Almeida, RS), 1737 (Maximiliano de Almeida, RS), 1738 (Maximiliano de Almeida, RS), 1763 (Maximiliano de Almeida, RS), 1769 (Maximiliano de Almeida, RS), 1771 (Maximiliano de Almeida, RS), EM-2108 (Cambará do Sul, RS), EM-2125 (Cambará do Sul, RS), LEM177 (sem registro de procedência), LEM-276 (sem registro de procedência), SNAP-002(sem registro de procedência). PNAS*: EM-1993, EM-2008.

Philander frenatus (Olfers 1818) $(\mathrm{n}=14)$

FLONA*: EM-3019. LEM: EM-1929 (Cambará do Sul, RS), GP-022 (Maquine, RS). MCNU: 124 (Barra do Ouro, RS), 128 (São Francisco de Paula, RS). PNAS*: EM-1928. SMARC*: RB001, RB-002, RB-003, RB-004, RB-005, RB-006. UNICAMP: 1943 (Capão Bonito, SP), 2386 (Ribeirão Preto, SP).

CÓDIGOS: ARA: Estação Ecológica de Aracuri - RS. FLONA: Floresta Nacional de São Francisco de Paula - RS. FZB: Fundação Zoobotânica do Rio Grande do Sul. LEM: Laboratório de Ecologia de Mamíferos - Universidade do Vale do Rio do Sinos - RS. $M C N U$ : Museu de Ciências Naturais da Ulbra - RS. PNAS: Parque Nacional dos Aparados da Serra RS. SMARC: São Marcos - RS. UnB: Museu de Zoologia da Universidade de Brasília. UNICAMP: Museu de Zoologia da Universidade de Campinas ZOO: Parque Jardim Zoológico de Sapucaia do Sul - RS. $s / n^{o}$ : animais sem número de tombo. * = animais capturados em campo e liberados - consta, portanto, o número de campo como identificação.

CODES: ARA: Estação Ecológica de Aracuri - RS. FLONA: Floresta Nacional de São Francisco de Paula - RS. FZB: Fundação Zoobotânica do Rio Grande do Sul. LEM: Laboratório de Ecologia de Mamíferos - Universidade do Vale do Rio do Sinos - RS. MCNU: Museu de Ciências Naturais da Ulbra - RS. PNAS: Parque Nacional dos Aparados da Serra RS. SMARC: São Marcos - RS. UnB: Museu de Zoologia da Universidade de Brasília. UNICAMP: Museu de Zoologia da Universidade de Campinas ZOO: Parque Jardim Zoológico de Sapucaia do Sul - RS. $s / n^{o}$ : animals without museum number. * = animals that were captured and released after collection of hair samples - thus the identification number is the field number. 
Anexo II. Chave dicotômica para identificação de marsupiais do Rio Grande do Sul através das características microscópicas dos pelos-guarda

Annex II. Dichotomous identification key for the marsupials of Rio Grande do Sul state based on microscopic characteristics of their guard hairs.

1 - Medula unisseriada na porção medial do escudo (Fig. 1b e 1f)

1' - Medula multisseriada na porção medial do escudo (Fig. 1a e 1d)

2 - Medula escalariforme na porção medial do escudo (Fig. 1f e 1i)

2' - Medula literácea na porção medial do escudo (Fig. 1b e 1g)

3 - Cutícula pavimentosa losângica na região medial da haste (Fig. 2b e 2g) Monodelphis gr. dimidiata.

3' - Cutícula imbricada conoidal ou folidácea na região medial da haste (Fig. 2e e 2f)

4 - Cutícula imbricada folidácea larga ou intermediária na região medial da haste (Fig. 2i) Caluromys lanatus.

4' - Cutícula imbricada conoidal na região medial da haste (Fig. 2d e 2f)

5 - Cutícula imbricada conoidal simétrica (o ápice do cone tem tamanhos similares entre os lados) na porção medial da haste (Fig. 2d) e losângica intermediária na porção final da haste (entre a haste e o escudo) .Gracilinanus agilis ou G. microtarsus.

5' - Cutícula imbricada conoidal assimétrica (o ápice do cone tem tamanhos diferentes entre os lados) na porção medial da haste (Fig. 2f) e ondeada na porção final da haste (entre a haste e o escudo) Micoureus paraguayanus.

6 - Cutícula imbricada folidácea intermediária na porção medial da haste (Fig. 2h) Philander frenatus.

6' - Cutícula pavimentosa losângica na porção medial da haste (Fig. 2b e 2g).

7 - Cutícula losângica sem um eixo central aparente na porção medial da haste (Fig. 2g); células da medula ocupando $3 / 5$ do tamanho da espessura do pelo na porção medial do escudo (Fig. 1i); córtex geralmente bastante escuro..... Monodelphis gr. dimidiata.

7' - Cutícula losângica, com um eixo central aparente na porção medial da haste (Fig. 2b); células da medula ocupando 1/2 do tamanho da espessura do pelo na porção medial do escudo (Fig. 1b); córtex claro, geralmente com tons amarelados..... .Cryptonanus guahybae.

8 - Cutícula imbricada folidácea estreita na porção medial da haste (Fig. 2e) . Lutreolina crassicaudata.

8' - Cutícula imbricada folidácea larga (Fig. 2i) ou ondeada na porção medial da haste (Fig. 2a e 2c) 9.

9 - Medula anastomosada reticulada na porção medial do escudo (Fig. 1a)

Chironectes minimus.

9' - Medula anastomosada não-reticulada na porção medial do escudo (Fig. 1c e 1d) 10.

10 - Medula crivada simples na porção medial do escudo (Fig. 1d) Didelphis aurita.

10' - Medula crivada trilobada (o pelo apresenta três lobos, dando a impressão de haver uma fileira de células passando sobre as demais) na porção medial do escudo (Fig. 1c) Didelphis albiventris. 